\title{
Importancia de la integridad de la mucosa y submucosa uretral en la continencia de los pacientes con esfinteres urinarios artificiales
}

\author{
García Montes F*,**, Mundy AR*, Knight SL*, Craggs MD*. \\ *Institue of Urology and Nephrology. University College of London. ${ }^{* *}$ Hospital Universitario Son Dureta, \\ Palma de Mallorca Islas Baleares.
}

Actas Urol Esp. 2007;31(5):482-487

\begin{abstract}
RESUMEN
IMPORTANCIA DE LA INTEGRIDAD DE LA MUCOSA Y SUBMUCOSA URETRAL EN LA CONTINENCIA DE LOS PACIENTES CON ESFINTERES URINARIOS ARTIFICIALES

Introducción: La reaparición de incontinencia por atrofia uretral en pacientes con esfinteres urinarios artificiales (EUA) tradicionalmente ha sido atribuida al déficit de transmisión de la presión oclusiva. Existe, sin embargo, una fuerza pasiva que reside en la mucosa y submucosa uretral y contribuye al sellado de la luz uretral. Nuestro objetivo fue determinar el papel de la mucosa y submucosa uretral en la continencia de los pacientes con EUA en la uretra bulbar.

Materiales y Métodos: Se estudiaron dos grupos de pacientes con un EUA in situ durante más de 1 año $\left(\mathrm{n}_{\text {total }}=11\right)$. El grupo I estaba constituido por pacientes continentes $\left(n_{I}=6\right)$ y el grupo II por pacientes incontinentes debido a atrofia uretral $\left(n_{I I}=5\right)$. Se valoró la presión intrauretral (PI) y la perfusión sanguínea (PS) de la mucosa y submucosa de forma simultánea con la técnica de microtransductor multicanal y láser-doppler respectivamente. El estudio se realizó comparando la PI y la PS de la uretra proximal y distal al manguito con la PI y la PS del segmento uretral por debajo del manguito en cada uno de los pacientes.

Resultados: La PS del grupo I (continente) no varió de forma significativa a lo largo de la uretra. La PS del grupo II (incontinente por atrofia uretral) fue significativamente menor por debajo del manguito que en la uretra proximal y distal. La IP del tramo uretral por debajo del manguito de ambos grupos no presentó diferencias significativas.

Discusión: La continencia de los pacientes con EUA depende de dos factores: 1) la presión que el manguito ejerce sobre la uretra bulbar y 2) la preservación de la perfusión sanguínea de la mucosa y submucosa uretral. Para un determinado rango de presiones en el manguito, el factor más importante es la perfusión sanguínea uretral. Los pacientes con buena perfusión permanecerán continentes y aquellos en los que la isquemia impide el efecto de sellado de la mucosa y submucosa desarrollarán incontinencia.
\end{abstract}

Palabras clave: Atrofia uretral. Esfinter urinario artificial. Incontinencia de orina.

\section{ABSTRACT}

THE ROLE OF LOW URETHRAL MUCOSAL AND SUBMUCOSAL BLOOD PERFUSION IN PATIENTS WITH ARTIFICIAL URINARY SPHINCTERS

Introduction: A common complication following insertion of a bulbar AUS is recurrent incontinence, and once other causes have been ruled out, a diagnosis of urethral atrophy is then made. Urethral atrophy probably occurs to a certain degree in all patients with an AUS but the reappearance of incontinence is often attributed to inadequate pressure transfer from the cuff to the atrophic urethra. The normal urethral closure mechanism depends also on passive forces which rely on the integrity of the urethral mucosa and submucosa.

Material and Methods: Two groups of patients with a first AUS implant which had been in place for more than 1 year were studied $(n=11)$. Group I consisted of continent patients $\left(n_{I}=6\right)$ whereas Group II had significant incontinence due to urethral atrophy $\left(\mathrm{n}_{\mathrm{II}}=5\right)$. Intraurethral pressures (IP) and blood flux $(\mathrm{BF})$ were measured simultaneously with a micro-tip transducer and a laser Doppler flowmeter in each patient. Positional measurements were recorded proximal, within, and distal to the cuff first with the AUS deactivated and then activated.

Results: Group I patients exhibited similar IP and BF at all positions along the urethra. In Group II the IP was similar along the urethra but the BF within the cuff was qualitatively different (non pulsatile) and decreased significantly when compared to either side of the cuff during.

Discussion: Continence in patients with artificial urinary sphincters depends not only on the cuff occlusive force but is also dependent on the viability of the mucosal and submucosal tissues. For a given pressure range mucosal and submucosal blood perfusion is determinant: patients with normal blood perfusion would remain continent whereas patients with impaired perfusion would become incontinent.

Keywords: Urethral atrophy. Artificial urinary sphincter. Urinary incontinence. 
Ciempre que se ejerce una presión concéntrica constante sobre un tejido se produce isquemia y, consecuentemente, atrofia de dicha estructura. Ello ocurre de forma casi invariable tanto a nivel del cuello vesical como de la uretra bulbar cuando se coloca el esfinter urinario artificial (EUA) AMS-800. No obstante, y debido al grosor del mismo, las implicaciones clínicas (isquemia, atrofia y erosión) en cuello vesical tras la implantación del manguito de un EUA son poco frecuentes, al menos en lo que se refiere a la etapa precoz. La atrofia de la uretra bulbar suele manifestarse como reaparición de la incontinencia en un periodo variable post-implante, típicamente a los 3 a 6 meses.

Siempre que se coloque un manguito que ejerza presión periuretral se producirá atrofia. Que la atrofia se manifieste clínicamente (reaparición de incontinencia) depende de varios factores, incluyendo el tamaño del manguito y la presión del reservorio. Tradicionalmente se asume que cuando la atrofia progresa hasta un punto crítico, el manguito deja de transmitir su presión a una uretra de menor calibre. El resultado, representado en la Figura 1, es un segmento uretral rígido y de diámetro reducido, sobre el que el manguito no puede ejercer suficiente presión. En esta situación no tiene sentido poner un reservorio de mayor presión y la única solución es cambiar el manguito por uno de menor tamaño o añadir un segundo manguito. No obstante, en esta explicación de la reaparición de incontinencia no se tienen en cuenta factores pasivos como ejercidos por la mucosa y submucosa uretral, cuya vascularización es crucial para ejercer su capacidad de sellado contribuyendo a la continencia. Nuestro objetivo fue el determinar el papel de la mucosa y submucosa uretral en el cierre de la luz uretral, y por tanto en la continencia de los pacientes con EUA en la uretra bulbar.

\section{PACIENTES, MATERIALES Y MÉTODOS}

Se estudiaron dos grupos de pacientes portadores de un EUA con el manguito colocado en la uretra bulbar durante más de 1 año (n total $=11$ ). Todos los pacientes de ambos grupos tenían un manguito de $4 \mathrm{~cm}$ de longitud y un reservorio de 61-70 cm de $\mathrm{H}_{2} \mathrm{O}$. Ningún paciente había tenido

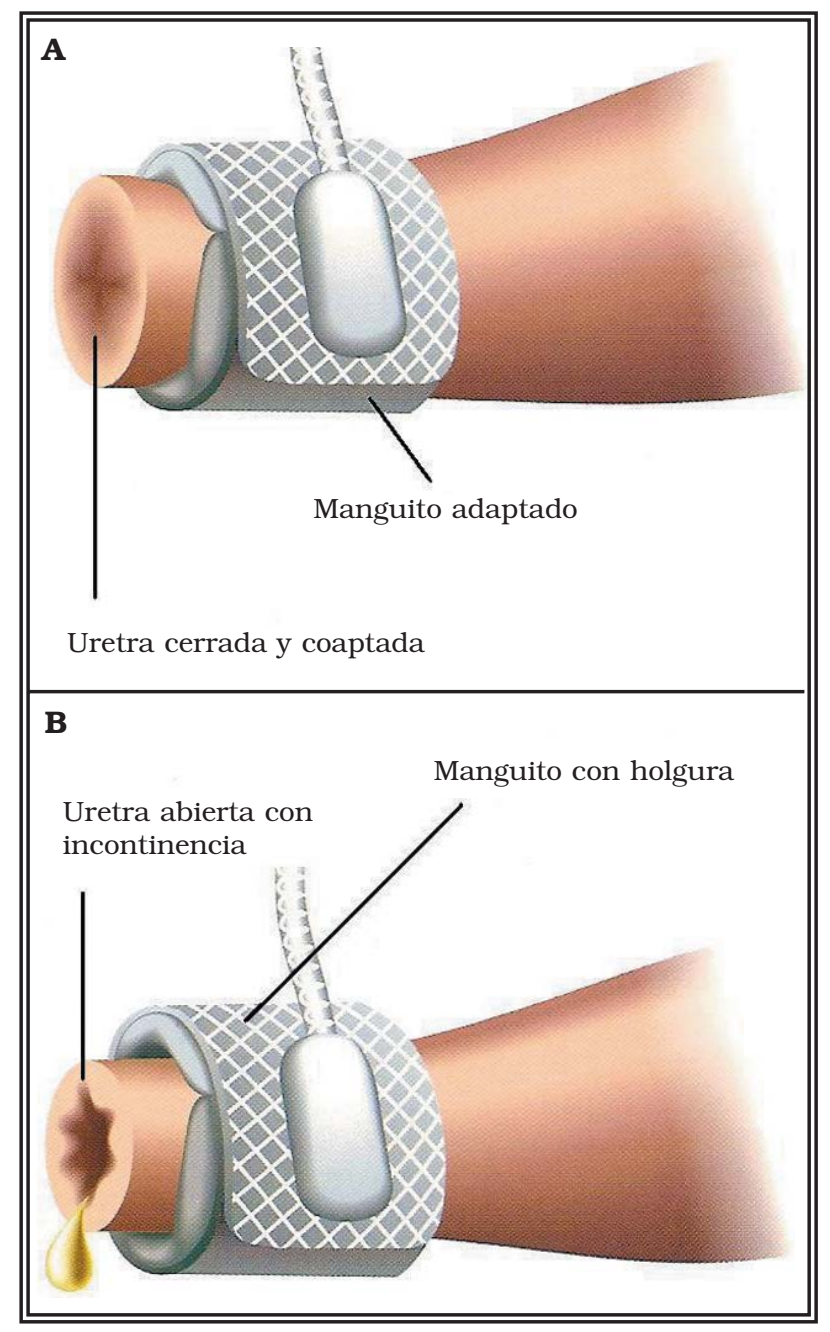

FIGURA 1. (A) Manguito del esfinter artificial AMS-correctamente adaptado a la uretra bulbar provocando oclusión de su luz y por tanto continencia. (B) Manguito con holgura por atrofia uretral en un paciente con incontinencia. Tradicionalmente la incontinencia se ha explicado por déficit de transmisión de presión oclusiva. En nuestro estudio se demuestra que, para cierto rango de presiones, la integridad de la mucosa y submucosa es un factor determinante para mantener la continencia en pacientes con atrofia uretral.

complicaciones perioperatorias ni había sido expuesto a radioterapia pélvica. El grupo I estaba constituido por pacientes continentes $\left(n_{I}=6\right)$ y el grupo II por pacientes incontinentes debido a atrofia uretral tras descartarse otras causas de reaparición de incontinencia por los métodos habituales $\left(\mathrm{n}_{\mathrm{II}}=5\right)$. Se valoró la presión intrauretral (PI) y la perfusión sanguínea (PS) de la mucosa y submucosa uretral de forma simultánea con la técnica de microtransductor multicanal y láser-doppler respectivamente. El estudio se rea- 
lizó comparando la PI y la PS de la uretra proximal y distal al manguito con la PI y la PS de la porción de la uretra por debajo del manguito en cada uno de los pacientes. Posteriormente se comparó la PI y la PS por debajo del manguito en el grupo de pacientes continentes con el grupo de pacientes incontinentes por atrofia uretral. Todas las mediciones se realizaron primero con el esfinter desactivado y luego con el esfinter activado.

\section{Perfil de presión intrauretral}

Las mediciones se realizaron mediante la técnica con microtransductor multicanal, basada en tecnología semiconductora avanzada. Dicha técnica reproduce los resultados obtenidos con la técnica de membrana pero consume menos tiempo al no requerir controles preoperativos, purgado del sistema o estabilizar presiones previas; además, el catéter es maleable por lo que facilita su introducción en la uretra de pacientes con esfinteres artificiales. Cada catéter de presión presenta dos chips cubiertos con silicona sensibles a los cambios de presión y cada chip contiene resistencias que convierten los cambios de presión en señales eléctricas, lo que permite obtener un trazado de las presiones intrauretrales. Mediante la manipulación de los transductores se puede obtener la presión intrauretral por debajo del manguito y las presiones intrauretrales proximales y distales al manguito del esfinter artificial. La Figura 2 representa la técnica y gráfica obtenidas durante la medición de presiones intrauretrales en un paciente con un esfinter urinario artificial en uretra bulbar.

\section{Flujometría intrauretral con láser-doppler}

El sistema de láser-doppler Periflux 4002 Satellite utiliza diodos que permiten la selección de determinadas longitudes de onda para proporcionar información sobre el estado del lecho vascular de los tejidos a diferentes profundidades. Ninguna técnica de láser-doppler proporciona valores absolutos, pero el sistema gráfico del equipo permite visualizar valores y tendencias del lecho vascular estudiado y su posterior análisis computerizado. Para el estudio de perfusión uretral se seleccionó el tipo de señal en modo "flux"

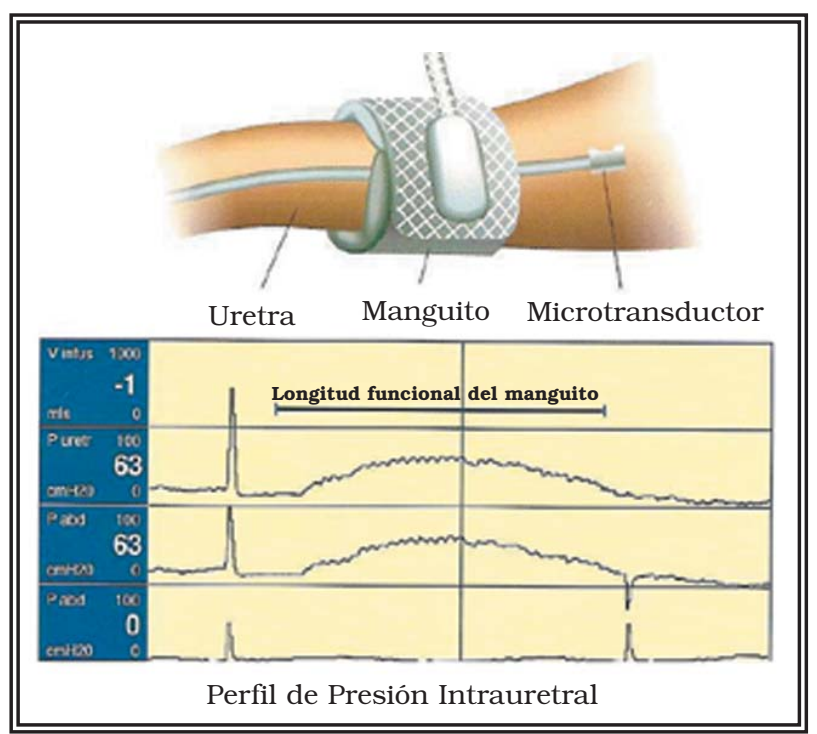

FIGURA 2. Técnica de medición de presión intrauretral con microtransductor multicanal en un paciente con el esfinter artificial AMS-800. En la gráfica se aprecia la longitud funcional del manguito con una presión intrauretral máxima de $63 \mathrm{~cm} \mathrm{H}_{2} \mathrm{O}$. El paciente portaba un reservorio de 61-70 $\mathrm{cm} \mathrm{H}_{2} \mathrm{O}$.

(perfusión de células sanguíneas) definido como el número de células sanguíneas en movimiento para un volumen dado multiplicado por la mediana de la velocidad de dichas células. El análisis cuantitativo se realiza en porcentaje de ascenso o descenso y el análisis cualitativo permite diferenciar un patrón pulsátil que refleja buena perfusión de un patrón lineal que refleja escasa perfusión. La Figura 3 representa la técnica y gráfica obtenida mediante la flujometría intrauretral con láser-doppler en un paciente con un esfinter artificial en uretra bulbar.

\section{Registro simultáneo de presión y perfución intrauretral}

El diseño de un catéter intrauretral maleable multicanal, dotado con microtransductores de presión y terminales láser-doppler, integrado en un programa computerizado de análisis de datos, permitió la obtención simultánea de registros de presión intrauretral proximal y distal al manguito así como del tramo uretral englobado por el manguito y la comparación de los resultados en los dos grupos de pacientes. Cada uno de los registros se realizó primero con el esfinter artificial desactivado y posteriormente se repitieron las mediciones con el esfinter activado. 


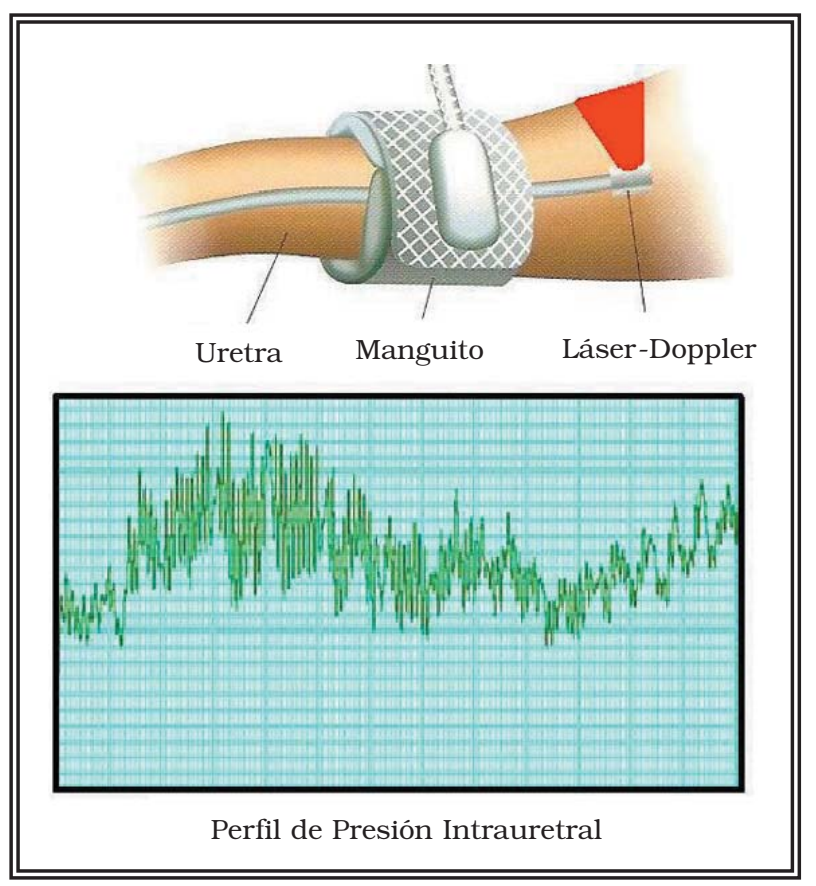

FIGURA 3. Flujometria intrauretral con láser-doppler utilizando el sistema Periflux 4002 Satellite en un paciente con un esfinter artificial en uretra bulbar. La gráfica recogida demuestra el patrón pulsátil típico de una uretra bulbar con buena vascularización de mucosa y submucosa. El paciente en cuestión portaba un AMS-800 con maguito de $4,5 \mathrm{~cm}$ y reservorio de $61-70 \mathrm{~cm} \mathrm{H}_{2} \mathrm{O}$ y mostraba continencia desde la fecha del implante.

\section{RESULTADOS}

La Figura 4 resume los resultados obtenidos. La perfusión sanguínea de la mucosa y submucosa uretral del grupo I (pacientes continentes) no varió ni cuantitativa ni cualitativamente (persistencia del patrón pulsátil) de forma significativa a lo largo de la uretra con el esfinter desactivado. Durante la activación, la presión intrauretral por debajo del manguito aumentó en menos del 35\% de la presión media del reservorio y la perfusión sanguínea de la mucosa y submucosa uretral descendió en menos de un 30\% sin modificación del patrón pulsátil.

La perfusión sanguínea de los pacientes pertenecientes al grupo II (pacientes incontinentes por atrofia uretral) fue significativamente menor e invariablemente presentó un patrón lineal en el tramo del manguito en comparación con la uretra proximal y distal, donde la perfusión era significativamente mayor y persistía el patrón pulsátil. La presión intrauretral del tramo uretral por debajo del manguito de ambos grupos no pre- sentó diferencias significativas. Durante la activación, la presión intrauretral por debajo del manguito aumentó en menos del 35\% de la presión media del reservorio (tal como ocurrió en el grupo I) pero la perfusión sanguínea de la mucosa y submucosa descendió en más del $45 \%$ persistiendo el patrón lineal.

\section{DISCUSIÓN}

A menudo se considera que la uretra es un conducto pasivo por el que discurre la orina. Sin embargo, el mecanismo de cierre uretral, y por tanto la continencia, depende de factores activos (la función del esfinter urinario) y pasivos (las características de la pared uretral). Para una determinada presión concéntrica adecuada sobre la uretra, la continencia depende de los factores mecánicos de la pared uretral presionada, fundamentalmente de la mucosa y submucosa ure$\operatorname{tral}^{1}$. Estos factores pasivos incluyen un comportamiento y capacidad de deformidad similar a los fluidos. La mucosa uretral está organizada en pliegues longitudinales que confieren una apariencia estrellada a la luz uretral cuando esta está cerrada, lo que se conoce con el nombre de "suavidad de la pared interna" y confiere la capacidad de coaptación y sellado. Dicha capacidad de sellado está asegurada por el rico plexo vascular que discurre por la submucosa uretral.

Desde un punto de vista simplista, la continencia urinaria se produce cuando la presión intrauretral es superior a la presión intravesical. La diferencia entre la presión intravesical y la presión intrauretral se conoce como presión de cierre uretral, pero el mecanismo por el que se mantiene la presión intrauretral sólo se conoce parcialmente. En primer lugar, el resto de la uretra presenta una presión superior a la de la vejiga, generalmente entre 40 y $80 \mathrm{~cm} \mathrm{H}_{2} \mathrm{O}$. Este rango de presión relativamente alto es debido a diferentes factores, algunos activos (acción del esfinter) y otros pasivos. Los factores pasivos incluyen la "suavidad de la pared interna" proporcionada por la mucosa y submucosa con su rica vascularización y la contribución de las fibras elásticas y de colágeno de la pared uretral. La uretra se puede asemejar a un cilindro, y la Ley de Laplace para un cilindro es Presión = Tensión / Radio. Por lo tanto, la elevada presión 


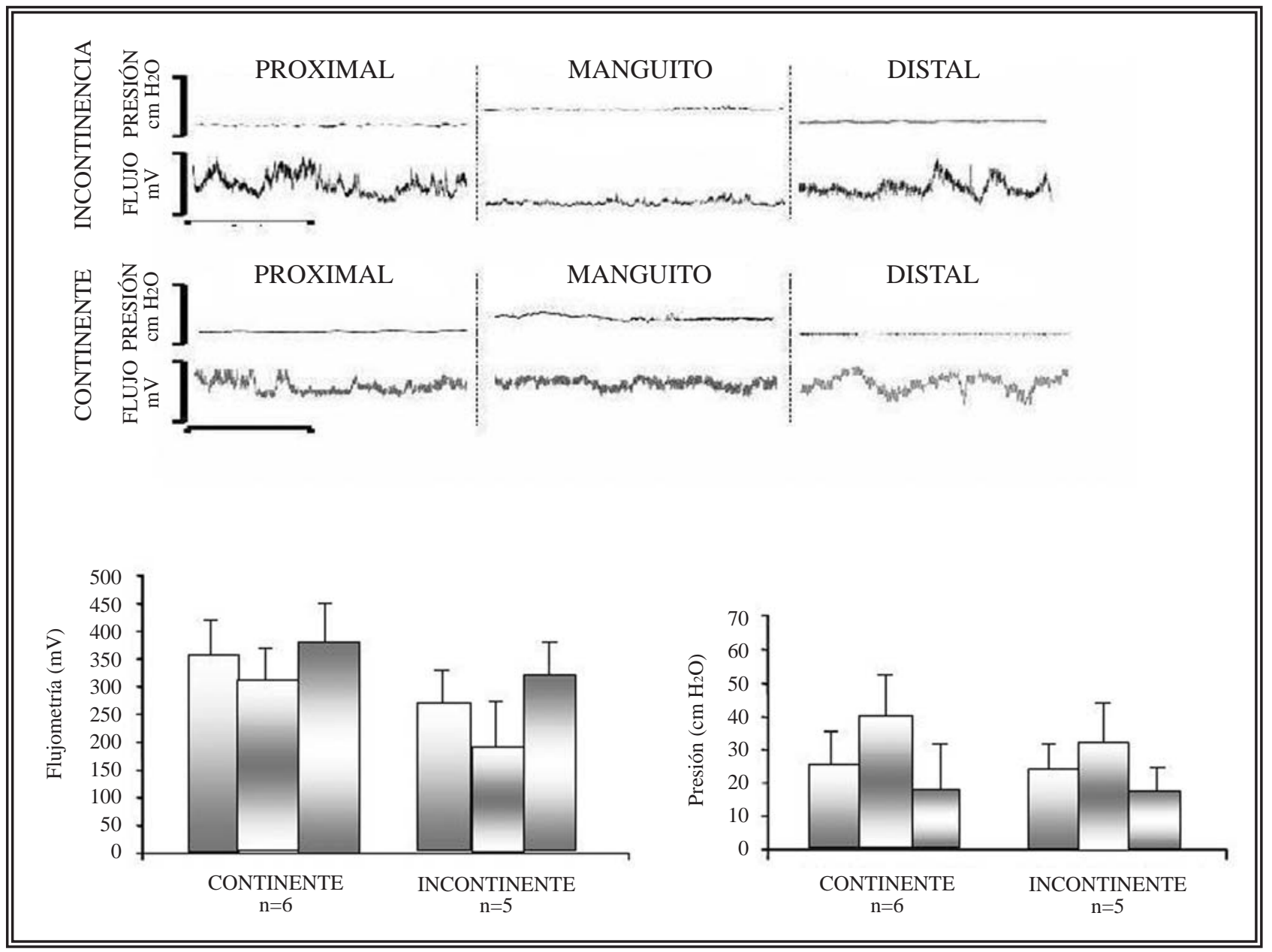

FIGURA 4. Resultados obtenidos mediante la medición de presión intrauretral con transductor multicanal y flujometría sanguínea con la técnica láser-doppler en pacientes con el esfinter AMS-800 en uretra bulbar. La porción superior de la imagen muestra los resultados en un paciente continente y uno incontinente. No existen diferencias significativas en la presión de oclusión, pero la perfusión de la mucosa y submucosa en la zona del manguito es cuantitativamente menor y presenta un patrón lineal en el paciente incontinente. En la porción inferior de la imagen se muestran los resultados en los dos grupos. Para presiones de oclusión similares, la única diferencia entre los pacientes continentes e incontinentes es la perfusión sanguínea del segmento uretral englobado por el manguito.

intrauretral es debida, al menos en parte, a la combinación de un radio pequeño con una elevada tensión (en comparación con el radio y tensión intravesical) ${ }^{1,2}$. En esta situación, la mucosa y submucosa uretral (y la gran irrigación que confiere sus propiedades de sellado) se convierten en el principal factor contribuyente de la reducción del calibre de la luz uretral y por tanto del radio.

Cuando se coloca el manguito de un esfinter artificial alrededor de la uretra bulbar, la presión ejercida por el mismo constituye la fuerza activa de cierre uretral. La complicación más frecuente es la reaparición de la incontinencia y cuando se han descartado otras causas se llega al diagnós- tico de incontinencia por atrofia uretral. La atrofia uretral, de hecho, se produce de forma casi invariable, sin embargo no todos los pacientes con atrofia uretral desarrollan incontinencia. Tradicionalmente se ha asumido que el mecanismo de la aparición de la incontinencia es secundario a la reducción del calibre de la pared uretral atrófica $y$, por tanto, por una inadecuada transmisión de presión a la pared uretral subyacente. Sin embargo, si se tienen en cuenta los factores arriba mencionados, sería tentador considerar esta explicación para la reaparición de la incontinencia un tanto simplista. De todos los factores que contribuyen al cierre uretral, el 
único que sufre cambios obvios entre la implantación de la prótesis y la reaparición de la incontinencia son la tensión de la superficie de la mucosa uretral y la vascularización de la submucosa. Con la atrofia de la mucosa y submucosa la "suavidad de la pared interna" y, por tanto, la capacidad de sellado desaparece. Blok et $\mathrm{al}^{3}$ demostraron en vejigas porcinas con esfinteres artificiales que la reaparición de la incontinencia tenía lugar con presiones intravesicales inferiores en al menos el $25 \%$ de la presión intrauretral ejercida por el manguito. Englemann ${ }^{4,5}$ estudió los efectos de manguitos presurizados en intestino animal demostrando la atrofia y devascularización de la mucosa y submucosa sin afectación de la pared muscular. Nuestros estudios en uretras humanas con esfinteres artificiales y las consideraciones previas sugieren que la integridad de la mucosa y submucosa uretral desempeñan un papel más importante que el previamente reconocido en el desarrollo de incontinencia por atrofia uretral. La existencia de pacientes con atrofia uretral pero sin incontinencia urinaria y con presiones intrauretrales similares a pacientes con atrofia uretral e incontinencia, hace sospechar que persiste la capacidad de coaptación de estas estructuras. De esta forma, la reaparición de la incontinencia no depende sólo de la presión que transmita el manguito (factor activo) sino que, para una presión dada, el factor determinante es el estado vascular de la mucosa y submucosa uretral $^{6-8}$. Para mantener la continencia, el esfinter urinario artificial AMS-800 ejerce elevadas presiones de oclusión de forma constante en la uretra, incluso en los periodos de reposo abdominal (la mayoría del tiempo) en los que no es necesaria tanta presión de oclusión. Dicha presión es la responsable de la mayoría de las complicaciones clínicas, incluyendo la atrofia uretral con o sin reaparición de incontinencia y erosión, que frecuentemente obligan a revisiones quirúrgicas.

\section{CONCLUSIONES}

La integridad de la mucosa y submucosa uretral parece poseer más importancia de la generalmente reconocida en la continencia de los pacientes con esfinteres urinarios artificiales con el manguito en la uretra bulbar. Lógicamente, cuando la presión de oclusión es baja el paciente presentará incontinencia y cuando dicha presión es alta el paciente estará continente. Existe, sin embargo un rango de presiones para los que la integridad de la mucosa y submucosa parece desempeñar un papel determinante. Las presiones de oclusión elevadas (superiores a $40 \mathrm{~cm}$ $\mathrm{H}_{2} \mathrm{O}$ ) desencadenan, tarde o temprano, atrofia uretral. La atrofia uretral implica una reducción de la transmisión de presión oclusiva desde el manguito a la uretra. Es precisamente en esta situación cuando la integridad de la mucosa y submucosa uretral determina la presencia o ausencia de incontinencia. Si las presiones de oclusión originales provocaron isquemia y pérdida de la capacidad de coaptación el paciente presentará incontinencia. Si la capacidad de coaptación persiste el paciente mantendrá la continencia.

\section{REFERENCIAS}

1. Zinner NR, Sterling AM, Ritter RC. Role of inner urethral softness in urinary continence. Urology 1980;16(1):115117.

2. Blaivas JG. The mechanism of micturition. In Williams DI, Chisholm GD, editors. Scientific Foundations of Urology. London, Heinemann Medical Books, 3 Edition. 1990, pp 273-285.

3. Blok C, Van Riel MP, Van Verooij GE, Coolsaet BL. Artificial sphincter pressure versus detrusor leakage pressure. J Urol 1985;133(1): 117-120.

4. Engelmann UH, Felderman TP, Scott FB. The use of the AMS-AS 800 artificial sphincter for continent urinary diversion. Investigations including pressure-flow studies, using rabbit intestinal loops. J Urol 1985;134(1):183-185.

5. Englemann UH, Felderman TP, Scott FB. Evaluation of the AMS-AS 800 artificial sphincter for continent urinary diversion using intestinal loops. Urology 1985; 25(6):620-621.

6. Knight S, Hussein I, Mundy AR, Craggs MD. The relationship between mucosal blood flow measured using laser Doppler flowmetry and pressure in the human penile urethra. Eur Urol 1998; Vol 33 (suppl 1): pp 97, Abstract 387.

7. Garcia Montes F, Knight S, Mundy AR, Craggs MD. The significance of low urethral blood flow in recurrent incontinence in patients with a long standing artificial urinary sphincter. Neurourol and Urodyn 1999;18(4):394-396.

8. Garcia Montes F, Knight S, Mundy AR, Craggs MD. Effects of artificial urinary sphincters on urethral blood perfusion measured with laser Doppler. Br J Urol Int 1999;8(1):154155.

Correspondencia autor: Dr. F. García Montes

Hospital Universitario Son Dureta

Andrea Doria, 55 - 07014 Palma de Mallora (Islas Baleares)

Tel.: 971175000

E-mail autor: fgmontes@wanadoo.es

Información artículo: Original - Urología funcional. Esfinter artificial

Trabajo recibido: noviembre 2006

Trabajo aceptado: marzo 2007 